\title{
Transatlantica
}

Revue d'études américaines. American Studies Journal

\section{Le Premier Amendement : un mythe}

\section{Claude-Jean Bertrand}

\section{(2) OpenEdition}

\section{Journals}

Édition électronique

URL : http://journals.openedition.org/transatlantica/545

DOI : 10.4000/transatlantica.545

ISSN : 1765-2766

Éditeur

AFEA

\section{Référence électronique}

Claude-Jean Bertrand, «Le Premier Amendement : un mythe », Transatlantica [En ligne], 1 | 2003, mis en ligne le 27 mars 2006, consulté le 29 avril 2021. URL : http://journals.openedition.org/ transatlantica/545; DOI : https://doi.org/10.4000/transatlantica.545

Ce document a été généré automatiquement le 29 avril 2021.

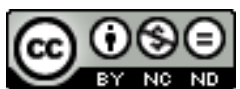

Transatlantica - Revue d'études américaines est mis à disposition selon les termes de la licence Creative Commons Attribution - Pas d'Utilisation Commerciale - Pas de Modification 4.0 International. 


\title{
Le Premier Amendement : un mythe
}

\author{
Claude-Jean Bertrand
}

\begin{abstract}
Premier amendement à la Constitution des Etats-
Unis, 1791 : « Le Congrès ne pourra faire aucune loi ayant pour objet l'établissement d'une religion ou interdisant son libre exercice, de limiter la liberté de parole ou de presse, ou le droit des citoyens de s'assembler pacifiquement et d'adresser des pétitions au gouvernement pour qu'il mette fin aux abus ».
\end{abstract}

1 Autrefois, un point d'interrogation eût été de rigueur, en fin de titre. Plus aujourd'hui. Nos cousins d'outre-Atlantique exagèrent à agiter sans cesse le Premier amendement comme un talisman. Un exemple: «The First Amendment is what distinguishes this Republic from all others in the world ", déclare Howard Simons, directeur de la Nieman Foundation, à Harvard. Et ils rêvent d'y convertir toute la planète !

2 Le Premier amendement constitue aux États-Unis une obsession quasi religieuse. Il me fait penser à ces sectes Étatsuniennes dont la théologie se fonde sur quelques versets obscurs de la Bible. Un signe de cette obsession? Le nombre d'ouvrages qui lui sont consacrés : plus de 60 livres dans les années 90 seulement, en ne comptant que ceux qui portent «Premier amendement » dans le titre et sont consacrés aux médias ${ }^{1}$. Il ne sera question ici que de la partie de l'amendement qui concerne la liberté de presse et de parole. C'est là que réside l'essentiel du problème.

3 N'étant pas spécialiste du droit de la presse, j'appliquerai au Premier amendement un gros bon sens. C'est là un comportement simpliste, il est vrai, mais il arrive des moments où il faut crier que le roi est nu. Il y a bien des années, j'ai découvert que, à proprement parler, le Premier amendement n'existait pas.

4 En septembre 2002, le First Amendment Center a fait faire une enquête d'où il ressort que 35\% des Étatsuniens ne peuvent pas nommer un seul des droits garantis par le Premier amendement ${ }^{2}$. Une enquête du Freedom Forum en $1997^{3}$ indiquait que 15\% seulement des Étatsuniens savaient qu'il y était question de liberté de presse. Si l'on considère que la grandeur des États-Unis repose sur la démocratie, que la démocratie 
repose sur la liberté d'expression et qu'aux États-Unis la liberté d'expression repose sur le Premier amendement, il peut paraître bizarre que la plupart des Étatsuniens l'ignorent.

5 En outre, on peut se demander si la minorité d'Étatsuniens qui connaît l'amendement le comprend, étant donné ce qu'elle en dit. Voici quelques citations qu'il faut lire en gardant à l'esprit le texte même de l'amendement: "Congress shall make no law... abridging the freedom of speech, or of the press ... $»^{4}$ :

Jane Kirtley, professeur de droit de la communication, dans sa chronique de janvier 2003 de l'American Journalism Review : « The First Amendment requires that truth must be a defense to any criminal libel charges, even if the statements were made with ill will or bad motives. »

Dans Broadcasting \& Cable du 18/12/95, après que les câblo-opérateurs US ont accepté qu'une puce (le V-chip) puisse limiter l'accès des enfants à la violence télévisée: "Having abdicated the First Amendment on the V-chip, the cable industry... »

En 1994, la municipalité de Chicago voulait déplacer le kiosques à journaux qui se trouvait en face de la bibliothèque municipale. Les kiosquiers ont invoqué la protection du Premier amendement, mais la US Court of Appeals ne l'a pas entendu de la même oreille. Néanmoins, en 1988, la Cour suprême avait décidé que les boîtes distributrices de journaux étaient protégées par le Premier amendement et que les municipalités ne pouvaient les déplacer ou les retirer à leur guise. ${ }^{5}$

Dans Editor \& Publisher du 19/4/86 : «A US judge in Tacoma (WA) recently ruled that a State statute banning exit polling within 300 feet of a voting place violated the First Amendment. »

Le 6 février 1985, le New York Times parlait d'un « procès intenté par quatre firmes de pornographie qui protestaient contre le plan de rénovation du quartier de Times Square, en vertu de leurs droits selon le Premier amendement. »

La même année, dans une lettre à Editor \& Publisher (23 mars), on lit : «Journalism has become an elitist profession no longer guided by the original ideals of the First Amendment. »

6 De toute évidence, ces gens ne parlent pas du véritable amendement, ce qui est compréhensible car ce qu'il dit est sans valeur. Il stipule que le Congrès (pas l'exécutif fédéral ou le judiciaire), juste le Congrès (et pas les législatures d'Etat ou les gouvernements locaux), ne fera aucune loi restreignant la liberté de parole ou de presse.

7 Or, à commencer par les Alien and Sedition Acts en 1798, le Congrès a fait des lois restreignant la liberté de parole et de presse, et même en grand nombre au $\mathrm{Xx}^{\mathrm{e}}$ siècle, tels le Postal Act de 1912 qui, entre autres, force les éditeurs à publier une fois l'an les noms des propriétaires des entreprises de presse et le chiffre des ventes; l'Espionage Act de 1917, utilisé contre la publication des Pentagon Papers en 1971. Surtout le Communications Act de 1934 et la Federal Communications Commission qu'il créait pour élaborer une réglementation de la radiotélévision et la faire respecter. La Cour suprême l'a accepté. Ou encore le Smith Act de 1940 contre la subversion communiste : dans l'affaire Dennis v. US (1951), la Cour suprême l'a jugé compatible avec le Premier amendement ; de même pour les lois anti-trust, dans l'affaire Associated Press, en 1945.

Une contradiction est apparue dès que les médias se sont commercialisés et que certains (comme la radiotélévision) se sont répandus hors de leur localité. Le conflit concerne le Premier amendement et l'Article 1 de la Constitution, clause 3, section 8, lequel déclare que « le Congrès aura le pouvoir de ... réglementer le commerce ... entre 
les divers États ». Et donc, depuis la naissance de l'industrie des médias, les lois fiscales, sociales, anti-monopole lui ont été appliquées comme aux autres industries.

Est-il stupide de prendre le Premier amendement à la lettre? Il y a eu au $x^{e}$ siècle d'éminents « absolutistes » du Premier amendement ${ }^{6}$ : Hugo Black, de la Cour suprême, affirmait que «pas de loi » voulait dire « pas de loi », un point, c'est tout - même en ce qui concerne les lois anti-monopole, comme il l'a soutenu (en vain) dans l'affaire de l'Associated Press.

10 En fait, dans tous les pays, une majorité s'accorde à penser que la liberté d'expression doit parfois céder à d'autres exigences de la société. Aux États-Unis comme ailleurs, on reconnaît l'importance de la sécurité nationale, d'où l'interdiction de paroles présentant «a clear and present danger »; l'importance pour chacun de sa réputation, et donc la nécessité de punir la diffamation; l'importance de protéger les enfants, notamment contre la pornographie: il va de soi qu'en ce haut lieu du puritanisme, l'obscénité n'est pas protégée par le Premier amendement, avec pour effet que des œuvres littéraires, comme le Ulysses de Joyce, ont été longtemps interdites. Et on reconnaît aussi la valeur des droits d'auteur, des marques déposées, de la vie privée, etc.

11 D'où l'absurdité du Premier amendement. Cette absurdité engendre des déclarations stupéfiantes de la part de juristes tel Justice Brennan, de la Cour suprême, qui dans l'affaire Roth v. U.S. (1957), a déclaré: "The unconditional phrasing of the First Amendment was not intended to protect every utterance ». Comme on était très gêné, on a introduit une distinction entre deux types de "parole ", celle qui est protégée et celle qui ne l'est pas. Pourquoi alors n'a-t-on pas introduit ce genre de distinction entre deux types de Congrès, deux types de lois, deux types de liberté. Ce n'est qu'en 1952 que la Cour suprême a placé le cinéma sous le bouclier de l'amendement : auparavant on n'y voyait qu'un divertissement commercial. Il a fallu attendre 1976 pour qu'elle a accordé une protection à la publicité (parole commerciale) alors qu'elle la lui avait expressément refusée en 1942.

Il est normal, bien sûr, qu'une Constitution d'avant la Révolution industrielle (1787) soit interprétée, et même amendée, pour être adaptée aux États-Unis post-industriels. Peu importent les intentions des Pères fondateurs : ils ont écrit un texte pour leur temps et ne pouvaient prévoir l'avenir. Songez à la prééminence qu'a acquise la Présidence au $\mathrm{xx}^{\mathrm{e}}$ siècle. D'ailleurs certains passages étaient si vagues qu'on a dû, dès le début du XIX siècle leur construire une interprétation, d'où un nouveau rôle pour la Cour suprême, à partir de l'arrêt Marbury v. Madison (1803).

13 Selon l'interprétation actuelle, on considère que le Premier amendement interdit à l'exécutif, au législatif et au judiciaire de limiter la liberté de presse ou d'expression au niveau fédéral et (en pratique, depuis les années 1920) au niveau des États et des localités. S'agit-il là d'une extension acceptable du sens originel ? Je ne le pense pas.

14 Prenez des équivalents de la Bill of Rights et voyez ce qu'ils disent de la liberté de parole et de presse : ainsi l'article 12 de la Déclaration des droits de l'état de Virginie (1776) déclare que : «the freedom of the press is one of the greatest bulwarks of liberty, and can never be restrained but by despotic governments. "

15 L'article 11 de la Déclaration des droits de l'homme publié par la Révolution française en août 1789 indique que : «La libre communication des pensées et des opinions est un des droits les plus précieux de l'homme; tout citoyen peut donc parler, écrire, 
imprimer librement, sauf à répondre de l'abus de cette liberté dans les cas déterminés par la loi ».

Et l'article 19 de la Déclaration universelle des droits de l'homme, adoptée en 1948 par les Nations-Unies pose que :

Tout individu a droit à la liberté d'opinion et d'expression, ce qui implique le droit de ne pas être inquiété pour ses opinions et celui de chercher, de recevoir et de répandre, sans considérations de frontières, les informations et les idées par quelque moyen d'expression que ce soit.

Elle dit ce que disent les interprètes du Premier amendement. Aussi, la concision de l'amendement serait-elle simplement due à un trait de caractère de l'auteur? Non, et on peut le prouver. Car il y a eu une première mouture du Premier amendement ${ }^{7}$. Voici ce qu'avait d'abord écrit James Madison :

«The people shall not be deprived or abridged of their right to speak, to write or to publish their sentiments; and the freedom of the press, as one of the great bulwarks of liberty, shall be inviolable. $»^{8}$

Ce texte est clair et global. On peut, ce me semble, en conclure que l'amendement qui fut voté (puis ratifié) constituait une restriction consciente: c'est bien du Congrès, institution nouvelle et dominante, qu'on se méfiait. Et les États qui le désiraient gardaient le droit de le censurer : ils l'ont gardé au moins jusqu'au $14^{\mathrm{e}}$ amendement $^{9}$, et en fait jusqu'aux années 1920.

Difficile, dans ces conditions, de justifier les interprétations « créatives » du $\mathrm{xx}^{\mathrm{e}}$ siècle.

Et ce d'autant plus que ce qu'on interprète n'est plus tant le texte original que ce qu'on croit y lire. Voici le processus : (1) Le Premier amendement établit la liberté de presse (ce qui est déjà fort discutable). (2) La presse a besoin d'être fabriquée et vendue, certes. (3) Donc les journalistes ont droit d'accès à l'information (réunions, archives d'organismes publics, par exemple). Par conséquent (4) les boîtes distributrices de journaux ne peuvent être retirées ou même déplacées.

20 A l'extrême, des interprètes découvrent dans le Premier amendement des droits étonnants. Le Professeur Jerome Barron a défendu une "théorie » séduisante : d'après lui, la liberté de parole et de presse n'a pas de sens dans une société de masse si le citoyen ne peut se faire entendre. D'où Barron déduit que le Premier amendement garantit l'accès des citoyens aux médias. Les experts jugent que Barron va trop loin mais il ne va pas tellement plus loin que bien d'autres.

21 Considérez une décision récente de la Cour suprême. Le Révérend Jerry Falwell, prédicateur de télévision et un des chefs de la droite religieuse, avait été présenté dans un dessin de Hustler, magazine pornographique, comme ayant été dépucelé par sa mère ivre dans une cabane de jardin ${ }^{10}$. Il s'est estimé diffamé. Selon la Cour (1988), il ne l'était pas : le Premier amendement exige un vigoureux débat social.

22 En réalité, ce qu'on appelle « First Amendment law » relève non pas de l'interprétation rationnelle mais de l'imagination créatrice. Le résultat n'a pas grand chose à voir avec le Premier amendement tel qu'il existe. Un jeu intéressant consisterait d'ailleurs à prendre d'autres passages de la Constitution et de les soumettre au même traitement.

23 Le Premier amendement me semble avoir trépassé il y a bien longtemps. Ce à quoi les gens croient de nos jours, est un mythe - un mythe correspondant à la première version de Madison. Un mythe est un mythe : il peut être puissant, certes, mais il reste flou. Les interprétations qu'on en donne varient selon l'environnement et selon les interprètes. 
24 Le seul interprète devrait être la Cour suprême, mais bien d'autres s'en mêlent. L'obscénité, par exemple, est définie par les autorités locales. La définition de la diffamation est laissée, dans une large mesure, aux jurys. Ce que la Cour suprême a autorisé dans l'affaire du Stanford Daily (1978), le droit pour la police de fouiller une salle de rédaction sans mandat, a été interdit quelque temps plus tard par le Congrès.

En conséquence, les limites de la liberté de parole et de presse sont variables aux États-Unis. La jurisprudence est si complexe et si grande la latitude des juges qu'on ne peut jamais être sûr de l'issue d'un procès de presse. Dans des périodes comme l'après Première Guerre mondiale ou l'époque McCarthy la liberté s'en trouve gravement menacée. Les gens de média, qui vénèrent le Premier amendement, ne sont jamais convenablement protégés par lui. Plus de journalistes sont envoyés en prison par un juge offensé aux États-Unis que dans tout autre pays démocratique.

26 Au contraire, un pays tel que la Suède, petit mais riche et très respectueux des droits de l'homme, possède une loi sur la presse précise et claire. Cette loi (qui date de 1949) plonge elle aussi ses racines dans le XvIII ${ }^{e}$ siècle et a été, de la même façon, intégrée dans la Constitution ${ }^{11}$. Pourquoi la citer ? Parce qu'elle résout à la satisfaction générale la plupart des problèmes auxquels se heurtent les médias des Etats-Unis : accès à l'information, protection des sources, procès de presse, etc.

On considère traditionnellement que le principal ennemi de la liberté de presse aux Etats-Unis est le pouvoir politique. D'ailleurs, au cours du dernier demi-siècle, le gouvernement s'est conduit normalement: il n'a pas montré un infini respect pour cette liberté. À preuve les Dossiers du Pentagone. Mais il faut se souvenir également que des gouvernements ont fait voter un Freedom of Information Act, des Open Records laws, des Open Meetings laws, des Shield laws, qui accordent de grands privilèges aux médias. Ils ont procédé à la déréglementation des médias électroniques (depuis le milieu des années 70). A quoi il faut ajouter des décisions judiciaires comme celle de la Cour Suprême dans l'affaire The New York Times v. Sullivan (limitant la diffamation) en 1964 et, 10 ans plus tard, lors du scandale du Watergate.

Pendant ce temps, qu'a fait le Big Business, pour protéger et promouvoir la liberté de parole et de presse ? Cette simple question a de quoi choquer des oreilles US. Qu'est-ce que les milieux d'affaires ont à faire avec la liberté de parole et de presse ? Un point, en tout cas, n'est pas discutable: le Big Business a partie liée avec la presse, écrite ou électronique. Les États-Unis sont la seule grande nation où tous les médias, avec quelques exceptions négligeables, sont commerciaux.

En conséquence, le but naturellement poursuivi par les patrons de médias est non pas de servir le public, mais de gagner de l'argent, encore plus d'argent tous les ans, la majeure partie sinon la totalité de leurs revenus provenant non pas des citoyens mais d'autres firmes commerciales. Quant à leur second objectif, il est de maintenir le statu quo, puisqu'il leur est favorable, comme aux annonceurs, gens d'affaires eux aussi. Outre qu'aucun d'eux n'est élu, qu'aucun n'a de comptes à rendre aux usagers, le problème vient de ce que les objectifs financiers et politiques des milieux d'affaires ne sont pas toujours compatibles avec la liberté de presse.

En minimisant les dépenses et en maximisant les revenus, les dirigeants de médias restreignent la liberté des journalistes à servir le public convenablement. Il faut savoir qu'aujourd'hui, malgré la crise, le profit moyen des médias US (20\%) est plus du double de celui des autres industries. Dans certains groupes de journaux, on licencie des 
journalistes quand le profit passe en dessous de $25 \%{ }^{12}$. La plupart des médias US fonctionnent comme des pompes à fric, pas comme des services publics. C'est la liberté d'entreprise qui leur importe, pas la liberté de parole. D'ailleurs, les médias commerciaux s'accommodent d'ordinaire assez bien des régimes fascistes.

31 Ce qui les gêne n'est pas, comme ils le prétendent, qu'on leur dicte quelques contenus, mais qu'on menace leur porte-monnaie en tentant de leur imposer des obligations telles que faire des émissions pour enfants $(1990)^{13}$; ou donner du temps d'antenne aux candidats à des élections (1995); ou limiter la violence ou interdire la publicité pour les boissons alcoolisées à la télévision (1994). Comme disait H.L. Mencken : «Hit in the moneybag, they suddenly become fanatical devotees of the Constitution».

Actuellement, alors que les télécommunications deviennent cruciales pour tout le monde, la concentration du contrôle des médias s'est terriblement accentuée. La restriction de la liberté de parole et de presse par de grosses firmes commerciales est plus grave que jamais. Quelques personnes peuvent décider ce qui sera ou ne sera pas publié. Pour savoir ce qui est passé sous silence, voyez, entre autres, la liste des «Ten Best-Censored Stories $»^{14}$. Et cherchez quelle proportion des cibles du journalisme d'enquête sont des firmes commerciales et non des services publics. Voyez ce que fournissent les médias US en matière d'information internationale, de culture, d'éducation.

Contre de telles restrictions à une liberté essentielle, que peut faire le Premier amendement? Absolument rien. Il ne s'applique pas aux censeurs privés. De là l'existence de "speech codes" sur les campus et toutes les règles "politiquement correctes » : on peut tout dire sauf ce qui risque de déplaire à la minorité X, Y ou Z. Ne fût-ce que pour cette raison, le Premier amendement est d'une utilité limitée. On pourrait le comparer à la Ligne Maginot, fortification impressionnante que les blindés nazis se sont contentés de contourner en passant par la Belgique.

Une lueur d'espoir est apparue du côté du public. Il est plus éduqué, mieux avisé. Une majorité hétérogène a compris qu'elle pouvait exiger que ses droits soient respectés : ceux des consommateurs, des femmes, des Noirs et d'autres minorités, des vieux, des handicapés ou des homosexuels. Pour se faire entendre, la plupart ont dû d'abord créer leurs propres médias, comme l'« underground press » des étudiants dans les années 60 . La plupart de ces médias ont été harcelés par la police et par les tribunaux, en dépit des droits que leur accordait prétendument le Premier amendement ${ }^{15}$. Et ils ont reçu peu de soutien des médias ordinaires, malgré l'attachement que ceux-ci proclament au Premier amendement. Pourtant, s'il est besoin de protéger un droit à la parole, c'est bien celui de qui est impopulaire.

Cette expérience a été l'un des facteurs qui ont engendré la méfiance du public envers les médias. Son hostilité a été confirmée au cours des trente dernières années par une multitude de sondages - sans que les médias la prennent vraiment au sérieux.

Quel rapport entre tout ceci et le Premier amendement? Eh bien, le public aux États-Unis, tout attaché qu'il soit à la liberté de parole et de presse, semble n'avoir pas le même concept de l'amendement que les médias. Pour commencer, le public semble croire que la liberté de presse lui appartient à lui et non à ceux qui possèdent les médias.

37 Une grande part des Étatsuniens ne sont pas favorables à la déréglementation des médias électroniques, pourtant elle n'a pas cessé depuis les années 80 au nom du 
Premier amendement ${ }^{16}$. Une cible centrale des dé-régulateurs était la Fairness Doctrine, formulée par la FCC, confirmée par le Congrès puis par la Cour suprême. Elle exigeait que sur les questions importantes, il y ait débat à la radio et à la télévision et que divers points de vue soient présentés. La plupart des usagers estiment que c'est là un droit du citoyen, que d'entendre au moins deux avis. Et bon nombre d'entre eux pensent même que de nouvelles lois devraient forcer les journaux à être équitables dans leur présentation des questions d'intérêt public.

Selon l'enquête menée par le First Amendment Center en septembre 2002, 42\% des Étatsuniens estiment que la presse a trop de liberté. En 1997, une enquête du Freedom Forum ${ }^{17}$ a indiqué que $65 \%$ d'entre eux jugeaient que la liberté de presse devait être restreinte dans certaines occasions. Ces gens s'opposent à la censure; approuvent le journalisme d'enquête et le rôle de chiens de garde qu'assument les médias - mais ils ont la sagesse de penser que la vraie liberté n'existe pas dans une jungle ; que la vraie liberté ne peut venir que de règles décidées démocratiquement et fermement appliquées. La totale dérégulation amène à une dictature de l'argent. Il semble que les usagers, bien que très ignorants du Premier amendement, sachent en tout cas cela.

Ils n'ignorent pas que les médias ne sont pas indépendants. Selon le sondage du Freedom Forum en 1997, 81\% jugeaient que l'information était souvent indûment influencée par des élus, 87\% par les grosses sociétés, 88\% par les patrons de médias, 88\% par les annonceurs, $91 \%$ par la cupidité des médias.

Dans les années 90 , on a commencé à s'inquiéter de leur impopularité : on les jugeaient trop puissants, trop arrogants, dévoués aux forces d'argent, on leur reprochait de négliger le service du public. Les jugements des tribunaux (sous la forme de dommages et intérêts énormes) se faisaient l'écho de l'opinion publique.

Que faire? Vu le statut de "révélation divine » que possède le Premier amendement, il n'est pas question de supprimer la partie qui concerne parole et presse. Pas question non plus de l'amender. De toute manière, à l'heure actuelle, les médias US disposent de bien plus de liberté qu'ils n'en utilisent jamais - ce qui rend incongrue leur obsession de défendre tous ces privilèges qui seraient inclus dans le Premier amendement. Puisqu'il faut interpréter le mythe, peut-être faudrait-il y chercher, non plus des droits supplémentaires pour les médias, mais pour les usagers, notamment celui d'être bien servis par les médias. On peut penser que les Pères fondateurs songeaient plutôt à ces droits là qu'aux bilans de News International, de Time-Warner, ou de General Electric.

\section{NOTES}

1. En janvier 2003, si l'on recherche "The First Amendment » sur Google par exemple, on obtient $2,7 \mathrm{~m}$. de réponses, contre $2 \mathrm{~m}$. seulement pour Georges W. Bush et 424000 pour Saddam Hussein.

2. Un sondage Gallup réalisé en décembre 1979 avait révélé que trois Étatsuniens sur 4 ne savaient pas répondre quand on leur demandait ce qu'était le Premier amendement et ce qu'il contenait, dont 6 sur 10 parmi les diplômés de l'enseignement supérieur. 
3. Voir Editor \& Publisher, 29/3/1997.

4. Texte complet du Premier amendement : « Le Congrès ne pourra faire aucune loi ayant pour objet l'établissement d'une religion ou interdisant son libre exercice, de limiter la liberté de parole ou de presse, ou le droit des citoyens de s'assembler pacifiquement et d'adresser des pétitions au gouvernement pour qu'il mette fin aux abus. »

5. Editor \& Publisher, $25 / 6 / 88$.

6. Par exemple, en 1988, une Free Press Association, composée d'absolutistes, comptait 300 membres.

7. Pour qui serait tenté d'attribuer un sens à la place qu'occupe le Premier amendement dans la Déclaration, il est à noter d'ailleurs qu'à l'origine il était le quatrième.

8. Cité par J. Herbert Altschull, Agents of Power (New York: Longman, 1984), 26.

9. Le $14^{\text {ème }}$ amendement à la Constitution (ratifié en 1868) dit, dans sa section $1:$ « No state shall make or enforce any law which shall abridge the privileges or immunities of citizens of the United States; nor shall any state deprive any person of life, liberty, or property, without due process of law; nor deny to any person within its jurisdiction the equal protection of the laws. "

10. Jugement confirmé un peu plus tard quand la Cour refusa d'examiner le recours de l'ultra féministe Dworkin contre le même magazine.

11. Et ne peut être modifiée sinon par deux votes du Parlement séparés par une élection générale.

12. Voir Leaving the Readers Behind: the Age of Corporate Newspapering, sous la direction de Gene Roberts (Fayetteville : University of Arkansas Press, 2001).

13. Par le Children's Television Act.

14. Établie annuellement depuis plus de 20 ans par des experts sous les auspices de Sonoma State University.

15. La Cour suprême a approuvé la censure par les autorités des journaux de lycéens et d'étudiants (1988)

16. The Speaker and the Listener: A Public Perspective on Freedom of Expression, sous la direction de J. Immerwahr et al. (New York: The Public Agenda Foundation, 1980.) George Gallup, « Whose First Amendment Is It Anyway? ».

17. Voir Editor \& Publisher, 29/3/1997.

\section{RÉSUMÉS}

Ce papier, plein de verve, d'humeur et d'humour, pose, comme sait si bien le faire l'auteur, des questions profondes sous une apparence paradoxale. Il a été présenté lors d'un colloque sur le Premier amendement organisé à l'Université Lumière-Lyon 2 les 17 et 18 janvier 2003. Certaines communications ont été publiées dans le volume XXIV, $\mathrm{n}^{\circ} 1$ (2003), «Le premier amendement : un modèle américain des libertés » (sous la direction de Vincent Michelot) de la Revue Tocqueville. 


\section{AUTEUR}

\section{CLAUDE-JEAN BERTRAND}

Claude-Jean Bertrand est professeur émérite à l'Université Paris 2 (Institut français de presse) après avoir enseigné à Strasbourg et Nanterre. C'est un spécialiste reconnu des médias, sur lesquels il a publié une vingtaine d'ouvrages, et tout particulièrement de la déontologie. Parmi ses nombreuses publications on citera : Media Ethics and Accountability Systems, New Brunswick (NJ) \& London, Transaction, 2000 (traduction de La déontologie des médias, Paris, PUF, 1977, ouvrage traduit en plus de 10 langues), et An Arsenal For Democracy : Media Accountability Systems, Cresskill (NJ), Hampton Press, 2003 (traduction de L'Arsenal de la démocratie, Paris Economica, 1999, aussi traduit au Brésil et au Japon (2002)). On pourra se reporter, pour ces questions, au site http:// www.presscouncils.org 